\title{
Excess H, Suppressed He, and the Abundances of Elements in Solar Energetic Particles
}

\author{
Donald V. Reames ${ }^{1}$
}

Received: 6 August 2019 / Accepted: 21 September 2019 / Published online: 22 October 2019

(C) The Author(s) 2019

\begin{abstract}
Recent studies of the abundances of $\mathrm{H}$ and $\mathrm{He}$ relative to those of heavier ions in solar energetic particle (SEP) events suggest new features in the underlying physics. Impulsive SEP events, defined by uniquely large enhancements of $\mathrm{Fe} / \mathrm{O}$, emerge from magnetic reconnection in solar jets. In small, "pure," shock-free, impulsive SEP events, protons with mass-to-charge ratio $A / Q=1$ fit the power-law dependence of element abundance enhancements versus $A / Q$ extrapolated from the heavier elements $6 \leq Z \leq 56$. Sometimes these events have order-of-magnitude suppressions of $\mathrm{He}$, even though $\mathrm{H}$ fits with heavier elements, perhaps because of the slower ionization of He during a rapid rise of plasma from the chromosphere. In larger impulsive SEP events, He fits, but there are large proton excesses relative to the power-law fit of $Z>2$ ions, probably because associated coronal mass ejections (CMEs) drive shock waves fast enough to reaccelerate the impulsive SEPs but also to sample protons from the ambient solar plasma. In contrast, gradual SEP events are accelerated by wide, fast CME-driven shock waves, but those with smaller, weaker shocks, perhaps quasi-perpendicular, favor impulsive suprathermal residue left by many previous jets, again supplemented with excess protons from ambient coronal plasma. In the larger, more common gradual SEP events, faster, stronger shock waves sample the ambient coronal plasma more deeply, overwhelming any impulsive-ion component, so that proton abundances again fit the same power-law distribution as all other elements. Thus, studies of the power-law behavior in $A$ / $Q$ of SEP element abundances give compelling new information on the varying physics of SEP acceleration and properties of the underlying corona.
\end{abstract}

Keywords Solar energetic particles $\cdot$ Solar system abundances $\cdot$ Coronal mass ejections · Solar flares

D.V. Reames

dvreames@umd.edu

1 Institute for Physical Science and Technology, University of Maryland, College Park, MD 20742-2431 USA 


\section{Introduction}

The Wind spacecraft has enabled studies of hundreds of solar energetic-particle (SEP) events in its highly productive 25 years of operation. All of these events are different. One most profound difference lies in the relative abundances of the elements in SEPs compared with corresponding abundances in the solar photosphere or corona. New abundances of $\mathrm{H}$ and He add information that is sensitive to the physics of particle selection and acceleration. Some elements in SEP events are preferentially selected for acceleration while others are preferentially scattered as they propagate away from the source.

Two primary sources of SEP events have been identified (e.g. Reames, 1988, 1995b, 1999, 2013, 2015, 2017a; Gosling, 1993). In the small "impulsive" SEP events, where acceleration has been traced to sites of magnetic reconnection in solar jets (Kahler, Reames, and Sheeley, 2001; Bučík et al., 2018a, 2018b), element abundances are enhanced as a power law in mass-to-charge ratio $A / Q$, increasing by a factor of $\approx 1000$ across the periodic table from $\mathrm{H}$ and $\mathrm{He}$ to $\mathrm{Au}$ and $\mathrm{Pb}$ (Reames and $\mathrm{Ng}$, 2004; Reames, Cliver, and Kahler, 2014a) probably because of the reconnection physics (e.g. Drake et al., 2009).

In contrast, in the large "gradual" SEP events (Lee, Mewaldt, and Giacalone, 2012; Desai and Giacalone, 2016), shock waves, driven by fast, wide coronal mass ejections (CMEs; Kahler et al., 1984; Lee, 1983, 2005; Zank, Rice, and Wu, 2000; Cliver, Kahler, and Reames, 2004; Gopalswamy et al., 2012), mostly sample ambient coronal material. This material differs from that in the photosphere by a factor that depends upon the first ionization potential (FIP) of the element. High-FIP (>10 eV) elements are neutral atoms in the chromosphere while low-FIP elements are ions that are preferentially enhanced by a factor of $\approx 4$, probably by the action of Alfvén waves (Laming, 2015; Reames, 2018a; Laming et al., 2019), when swept up into the corona and later sampled as SEPs (Webber, 1975; Meyer, 1985; Reames, 1995a, 2014). After acceleration, ion scattering, depending upon magnetic rigidity, hence upon $A / Q$ at a given velocity (Parker, 1963; Ng, Reames, and Tylka, 1999, 2001, 2003, 2012; Reames, 2016a, 2019c), can also lead to power-law dependence, i.e. since Fe scatters less than $\mathrm{O}$, for example, $\mathrm{Fe} / \mathrm{O}$ is enhanced early but depleted later in an event, producing power-law enhancements that increase and then decrease with $A / Q$ (e.g. Breneman and Stone, 1985). The seed population sampled by shock waves often contains remnant ions pre-accelerated in impulsive events (Tylka et al., 2001; Mason, Mazur, and Dwyer, 2002); these impulsive seed particles can even dominate in $\approx 25 \%$ of gradual SEP events (Reames, 2016a).

The dependence upon $A / Q$, and thus upon $Q$, is a dependence upon the source-plasma electron temperature $T$, which allows us to estimate $T$ in a large sample of events, both impulsive (Reames, Meyer, and von Rosenvinge, 1994; Reames, Cliver, and Kahler, 2014b, 2015), where $T \approx 3 \mathrm{MK}$, and gradual (Reames, 2016a, 2016b), where $0.8 \leq T \leq 4 \mathrm{MK}$. The technique for making such estimates, by best-fitting enhancements versus $A / Q$ for many values of $T$ and selecting the one with minimum $\chi^{2}$, has been described in review articles (Reames, 2018b) and even textbooks (Reames, 2017a).

Thus it is common for SEP events of both types to show a power-law dependence on $A / Q$ of observed element abundances divided by the corresponding reference SEP-coronal abundances listed in the Appendix. It is these reference abundances that differ from solarphotospheric abundances because of the "FIP-effect" (e.g. Meyer, 1985; Reames, 1995a, 2014). The coronal abundances deduced from SEPs differ from those found for solar wind (Mewaldt et al., 2002; Desai et al., 2003; Kahler, Tylka, and Reames, 2009; Reames, 2018a; Laming et al., 2019). Current theory (Laming, 2015; Laming et al., 2019) suggests that the FIP-effect seen by SEPs begins on closed magnetic loops where Alfvén waves can resonate 
with the loop length, while that of the solar wind occurs on magnetic field lines that are open near the chromosphere-coronal boundary (Reames, 2018a; Laming et al., 2019). Thus SEPs are not accelerated solar wind.

Now we come to the elements $\mathrm{He}$ and $\mathrm{H}$. None of the early studies of $A / Q$-dependence of SEP abundances included $\mathrm{H}$, and many omitted He as well. Recent studies have shown that the source abundance of $\mathrm{He} / \mathrm{O}$ can vary by a factor of $\approx 2$ in gradual SEP events (Reames, $2017 b, 2018 b)$ but there are occasionally tenfold suppressions in impulsive SEP events (Reames, 2019a). Theoretically, suppression of He may result from its slow ionization, because of its uniquely high FIP of $24.6 \mathrm{eV}$, as it traverses the chromosphere (Laming, 2009). But how does this produce such large suppressions?

In contrast, H/O shows a very different behavior. In small impulsive SEP events (Reames, $2019 \mathrm{~b}$ ), $\mathrm{H}$ fits well onto the extension to $A / Q=1$ of the power-law fit of enhancements versus $A / Q$ for the elements with $Z \geq 6$, independently of whether He also fits. For larger impulsive events, and especially those associated with faster, but characteristically narrow, CMEs, the enhancement of $\mathrm{H}$ can exceed that fit line by an order of magnitude or more - a significant proton excess. Gradual SEP events also fall into two groups (Reames, 2019c). For those associated with ambient coronal temperatures of $0.8-1.5 \mathrm{MK}, \mathrm{H}$ generally falls close to the fit line from the elements with $Z \geq 6$ extrapolated down to $A / Q=1$. This applies to about $60 \%$ of gradual events. However, for those gradual SEP events with $T \approx 3 \mathrm{MK}$, which all have positive power-law slopes for $Z \geq 6$, presumably derived from shock-reaccelerated impulsive-SEP material, $\mathrm{H}$ again exceeds the extended fit line by an order of magnitude or more - a factor-of-ten proton excess.

Why do both impulsive and gradual events have subsets with a strong proton excess? Both of these subsets have element enhancements that increase with $A / Q$ and involve shock reacceleration of impulsive-SEP ions. To what extent do these subsets involve different physics? Have we merely made some mistake where we draw the line between impulsive and gradual events? Have we misidentified events? In this article we attempt to display and contrast those properties of the events that help disentangle our understanding of the physics of the relative abundances of $\mathrm{H}$ and $\mathrm{He}$ in all types of SEP events. What is the overall picture? How do the excesses and suppressions fit in?

Element abundances in this article were measured by the Low-Energy Matrix Telescope (LEMT) onboard the Wind spacecraft, near Earth (von Rosenvinge et al., 1995; see also Chapter 7 of Reames, 2017a). LEMT measures elements from $\mathrm{H}$ through $\mathrm{Pb}$ in the region of 2-20 MeV amu${ }^{-1}$, although energies of $\mathrm{H}$ are limited to 2-2.5 MeV and LEMT resolves only element groups above Fe as shown by Reames (2000, 2017a). We reexamine the impulsive events listed and studied by Reames, Cliver, and Kahler (2014a, 2014b) and the gradual events listed and studied by Reames (2016a). CME data used are observed by the Large Angle and Spectrometric Coronagraph (LASCO) onboard the Solar and Heliospheric Observatory (SOHO) as reported in the SOHO/LASCO CME catalog (Gopalswamy et al., 2009; https://cdaw.gsfc.nasa.gov/CME_list/).

\section{Impulsive SEP Events}

The uniqueness of impulsive SEP events was first recognized by the unusual abundance of ${ }^{3} \mathrm{He}$. Ratios of ${ }^{3} \mathrm{He} /{ }^{4} \mathrm{He}$ as high as $1.5 \pm 0.1$ (e.g. Serlemitsos and Balasubrahmanyan, 1975) contrasted with $5 \times 10^{-4}$ in the solar wind. Since the ${ }^{3} \mathrm{He}$ was completely unaccompanied by ${ }^{2} \mathrm{H}$ or the elements $\mathrm{Li}, \mathrm{Be}$, and $\mathrm{B}$, the ${ }^{3} \mathrm{He}$ had nothing to do with nuclear fragmentation, rather with a resonance process (e.g. Temerin and Roth, 1992) that was associated 
Figure 1 Histogram distinguishing impulsive and gradual SEP events shows the occurrence rate of various values of abundances $\mathrm{Ne} / \mathrm{O}$ versus $\mathrm{Fe} / \mathrm{O}$ in 20 years of 8-hr average measurements from the Wind spacecraft. Values which form the centers for selection of impulsive and gradual SEP events by Reames, Cliver, and Kahler (2014a) and Reames (2016a) are indicated. Events defined by $\mathrm{Fe} / \mathrm{O}$ in this way distinguish meaningful physical processes such as those of associated CMEs.

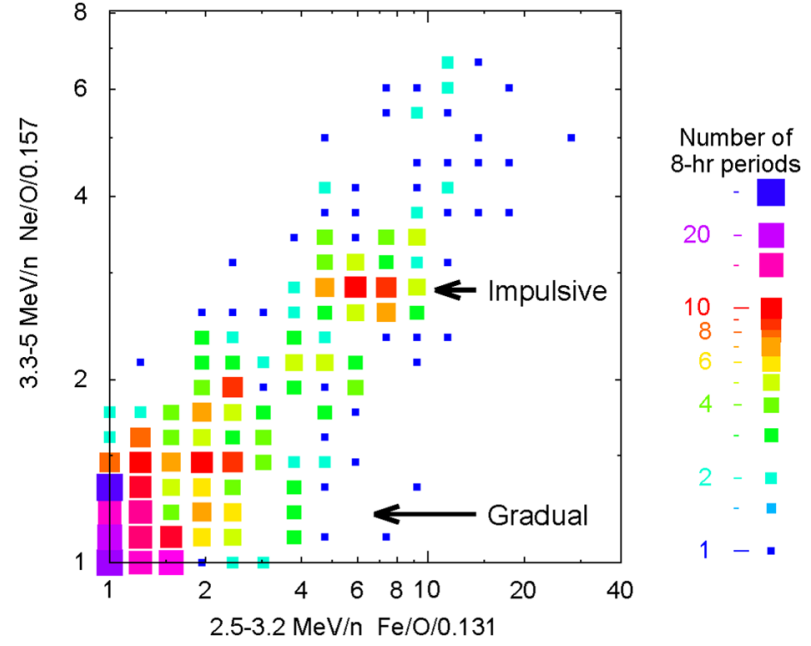

with streaming electrons and radio type III bursts (Reames, von Rosenvinge, and Lin, 1985; Reames and Stone, 1986). Element enhancements up to Fe (e.g. Reames, Meyer, and von Rosenvinge, 1994; Mason, 2007) and subsequently to heavier elements (Reames, 2000; Mason et al., 2004; Reames and $\mathrm{Ng}, 2004)$ were apparently accelerated directly in islands of magnetic reconnection (e.g. Drake et al., 2009).

However, since ${ }^{3} \mathrm{He} /{ }^{4} \mathrm{He}$ varies widely with energy in single events or from event to event (Mason, 2007), while Fe/O exhibits a bimodal distribution with impulsive events enhanced (Reames, 1988), Reames, Cliver, and Kahler (2014a) chose to define impulsive SEP events based upon $\mathrm{Fe} / \mathrm{O}$. The distribution in $\mathrm{Ne} / \mathrm{O}$ versus $\mathrm{Fe} / \mathrm{O}$ near $2.5 \mathrm{MeV} \mathrm{amu}^{-1}$ is shown in Figure 1. We define impulsive SEP events as having an abundance of at least four times the reference abundance of $\mathrm{Fe} / \mathrm{O}$ in the $2.5-3.2 \mathrm{MeV} \mathrm{amu}^{-1}$ energy interval.

Figure 2 shows properties of two impulsive SEP events that help to illustrate the concepts of "proton excess" and "He suppression". Throughout this article, He without a superscript always means ${ }^{4} \mathrm{He}$. Protons in Event 79 barely rise above the pre-event background, yet they do not exceed the power-law fit from the ions with $Z \geq 6$. Of the 111 impulsive SEP events studied (Reames, Cliver, and Kahler, 2014a), 70 have proton intensities above background and $17(24 \%)$ of these have proton intensities within one standard deviation of the value predicted by the best-fit line of the ions with $Z \geq 6$ (Reames, 2019a). Of these 17 events, 6 have order-of-magnitude He suppression. None of the larger events with proton excesses have significant He suppression.

At a temperature of $3 \mathrm{MK}$, He and $\mathrm{C}$ are fully ionized with $Q=Z$ and $\mathrm{O}$ is nearly so. Relative abundances of these ions with $A / Q \approx 2$ should remain unaltered during acceleration and transport. Figure 3 shows panels of O/C versus $\mathrm{He} / \mathrm{C}$ with $<20 \%$ errors, with various quantities represented in the color and size of the points. The scatter in the points is quite large, especially in the $\mathrm{He} / \mathrm{C}$ direction. Normalization based upon reference values of $\mathrm{He} / \mathrm{O}=57$ and 91 are shown as dashed lines. Various panels show the distribution of temperature, proton excess, peak proton intensity, and CME speed. It is clear that the proton excess is associated with events with higher $\mathrm{He} / \mathrm{C}$, with high proton intensities, and with fast CMEs, as noted by Reames (2019a). CMEs with speeds above $500 \mathrm{~km} \mathrm{~s}^{-1}$ are quite capable of ion acceleration. Smaller events do not have proton excess, but may have suppressed $\mathrm{He}$ $=57$ and 91. Note that the larger events with faster CMEs have higher average He/O. 

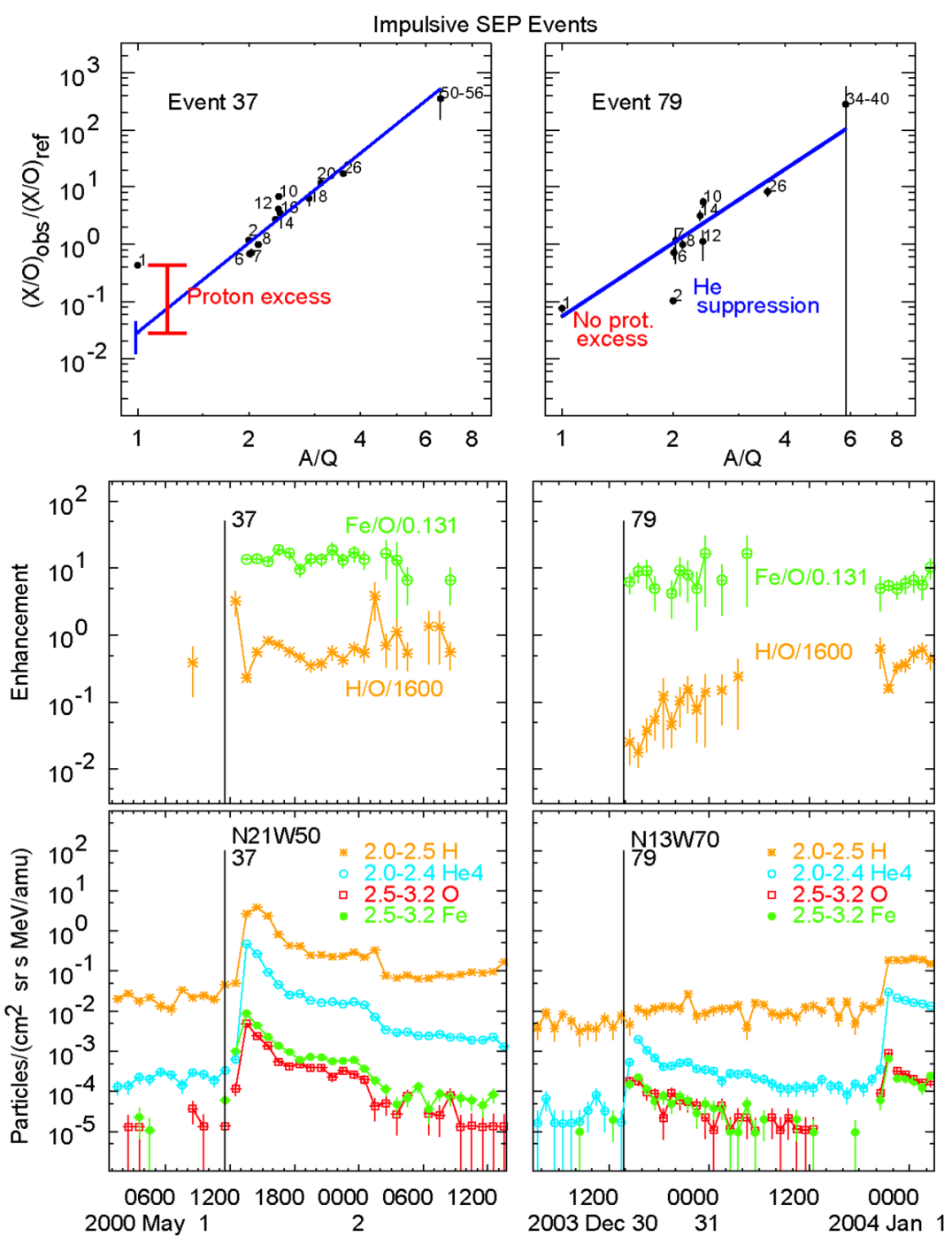

Figure 2 Intensities (lower panels), abundance ratios (middle panels) and enhancements versus A/Q (upper panels) are compared for two impulsive SEP events. Event numbers shown refer to the list of Reames, Cliver, and Kahler (2014a). Event 37 has a proton excess. Event 79 has no proton excess but has a large suppression of He.

\section{Gradual SEP Events}

Gradual SEP events are best characterized by their strong association with wide, fast CMEs (Kahler et al., 1984; Cane, Reames, and von Rosenvinge, 1988; Reames, Barbier, and Ng, 1996; Gopalswamy et al., 2012; Rouillard et al., 2012; Desai and Giacalone, 2016), an association that is extended by strong CME correlations (Kahler, 2001; Kouloumvakos et al., 2019) and by studies of associated radio type II bursts (e.g. Cliver, Kahler, and Reames, 2004). 
Figure 3 Each panel shows normalized abundances of $\mathrm{O} / \mathrm{C}$ versus $\mathrm{He} / \mathrm{C}$ with errors $<20 \%$ for impulsive SEP events with temperature, proton excess, $2 \mathrm{MeV}$ proton intensity, and associated CME speed highlighted by color and size of the points, as indicated. Dashed lines indicate reference abundances of $\mathrm{He} / \mathrm{O}$.

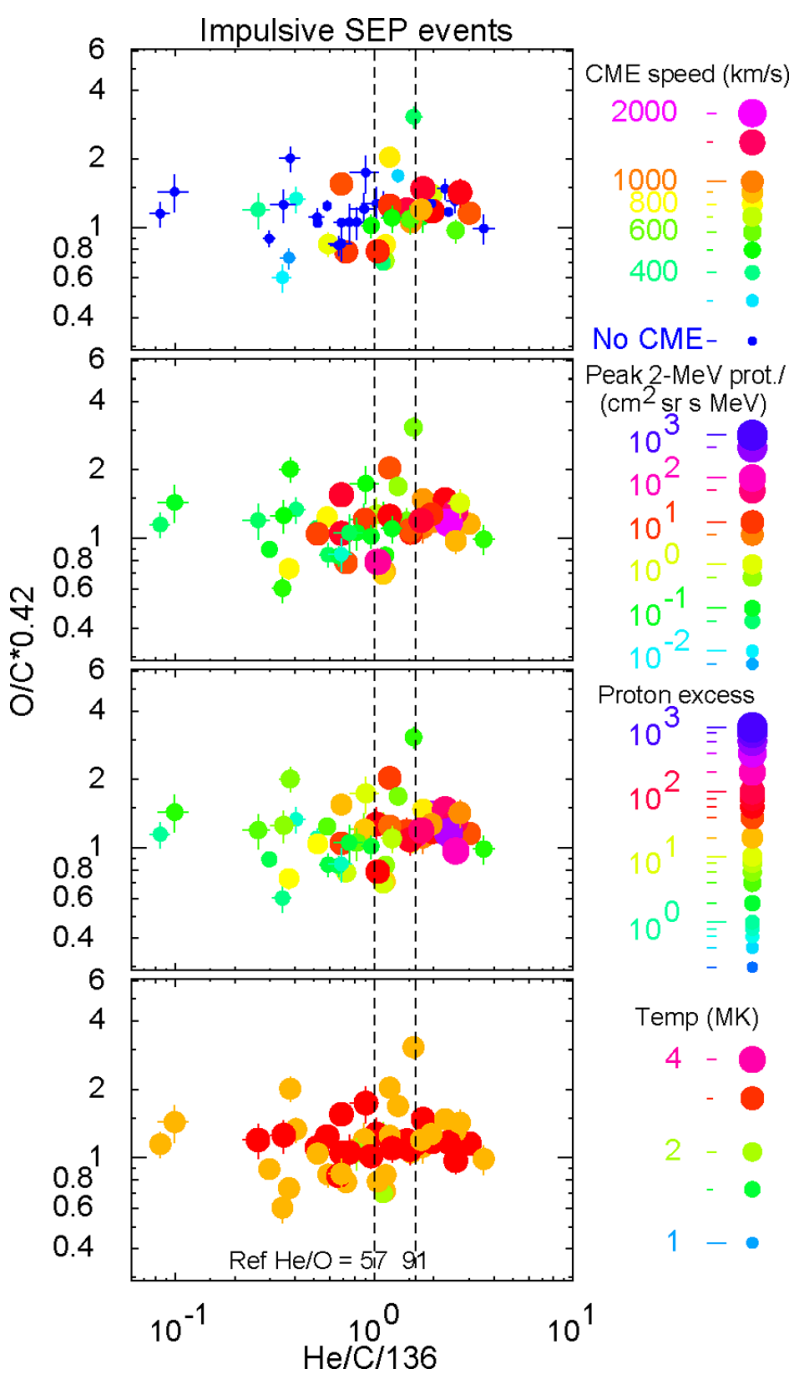

It is important to emphasize that most gradual SEP events do not show proton excesses. These are events with $T<2 \mathrm{MK}$. A new example is shown in Figure 4 and others are shown by Reames (2019c). Gradual SEP events are analyzed in 8-hr intervals since the abundances often change with time. The best power-law fits are shown for each time interval in the lowerright panel (e) of Figure 4 and the corresponding $\chi^{2}$ values that select them are shown (color coded) in the upper-right panel (d). It is not surprising that the fit to protons is occasionally broken, as in the second interval (14 September 2005 at 0000 UT) in Figure 4e, by spatial variations and scattering in the transport path, for example.

A clear and persistent proton excess is shown for the event in Figure 5. Power-law distributions for additional gradual SEP events have been shown by Reames (2016a).

The slope of the power law fitting the enhancements of $Z \geq 6$ ions tends to decrease with time. For the event in Figure 5 this power decreases from $1.9 \pm 0.2$ to $0.44 \pm 0.35$ during 

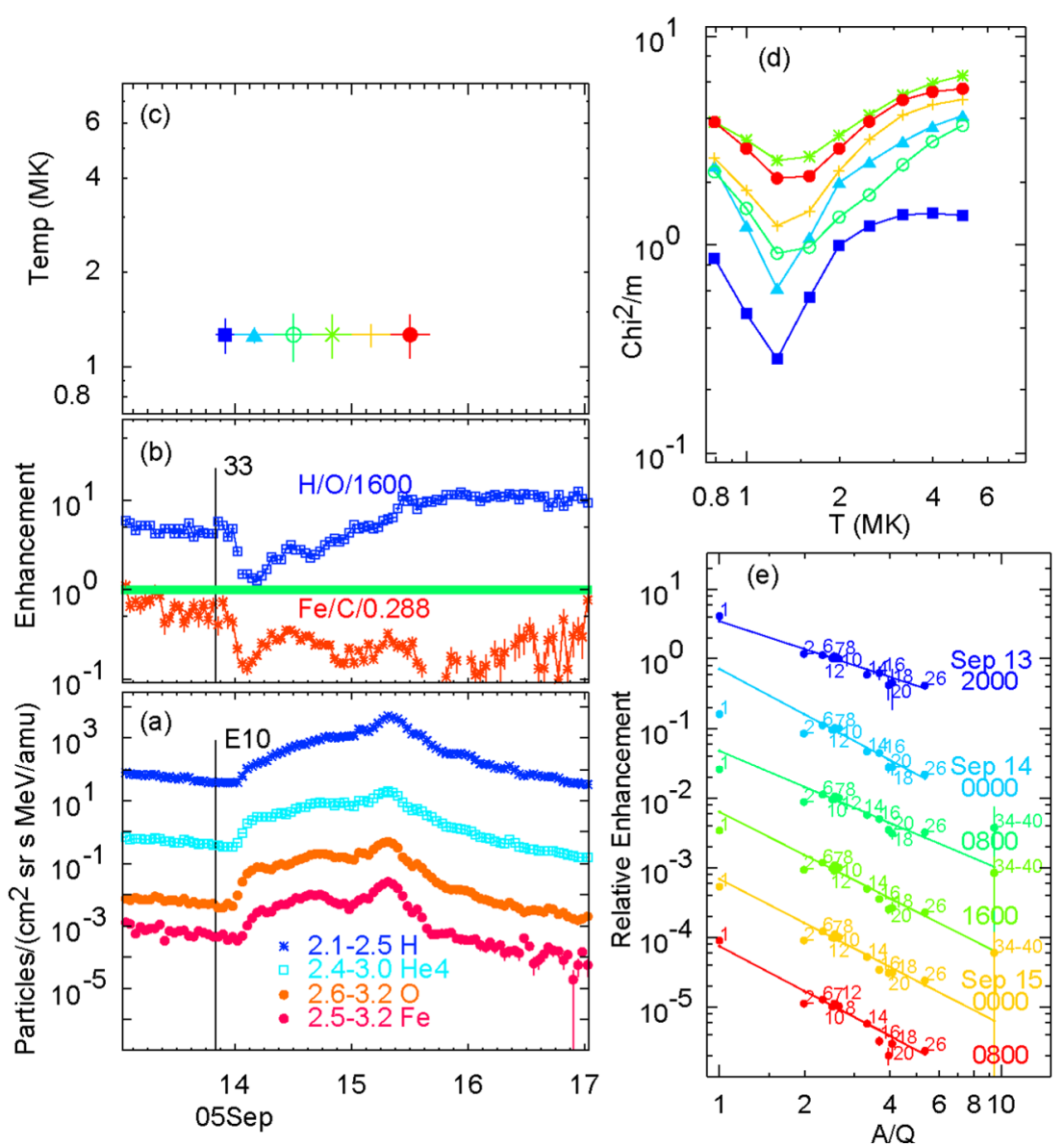

Figure 4 (a) Intensities of $\mathrm{H}, \mathrm{He}, \mathrm{O}$, and Fe versus time. (b) Normalized abundance enhancements $\mathrm{H} / \mathrm{O}$ and $\mathrm{Fe} / \mathrm{O}$ versus time. (c) Best-fit temperatures are shown versus time for the 13 September $2005 \mathrm{SEP}$ event. (d) Shows $\chi^{2} / m$ versus $T$ for each 8 -hr interval. (e) Shows enhancements, labeled by $Z$, versus $A / Q$ for each 8 -hr interval shifted $\times 0.1$, with best-fit power law for elements with $Z \geq 6$ extrapolated down to $\mathrm{H}$ at $A / Q=1$. Colors correspond for the six intervals in (c), (d), and (e) and symbols in (c) and (d); times are also listed in (e). Event onset is flagged with solar longitude in (a) and event number 33 from Reames (2016a) in (b). In most intervals in (e), protons fit the power law from higher $Z$.

the five eight-hour periods. During the final period it becomes so flat that $\chi^{2}$ also becomes flat and the temperature becomes uncertain.

The lower panel of Figure 6 shows O/C versus He/C for gradual SEP events at the same scale as it was shown for impulsive SEP events in Figure 3. In this case the scale leaves considerable white space because of the tighter grouping of gradual SEP events in O/C versus $\mathrm{He} / \mathrm{C}$.

The upper panels in Figure 6 show the tighter grouping of orange and red events with $T \approx 3 \mathrm{MK}$ in the He-rich, Fe-rich corner of the temperature panels. Big intense events, as measured by $20-\mathrm{MeV}$ protons and associated with the fastest CMEs (e.g. Kahler, 2001; Kouloumvakos et al., 2019), lie along the He-poor side and drift down in Fe/O with time. Thus acceleration in the largest gradual SEP events is dominated by ambient coronal plasma 

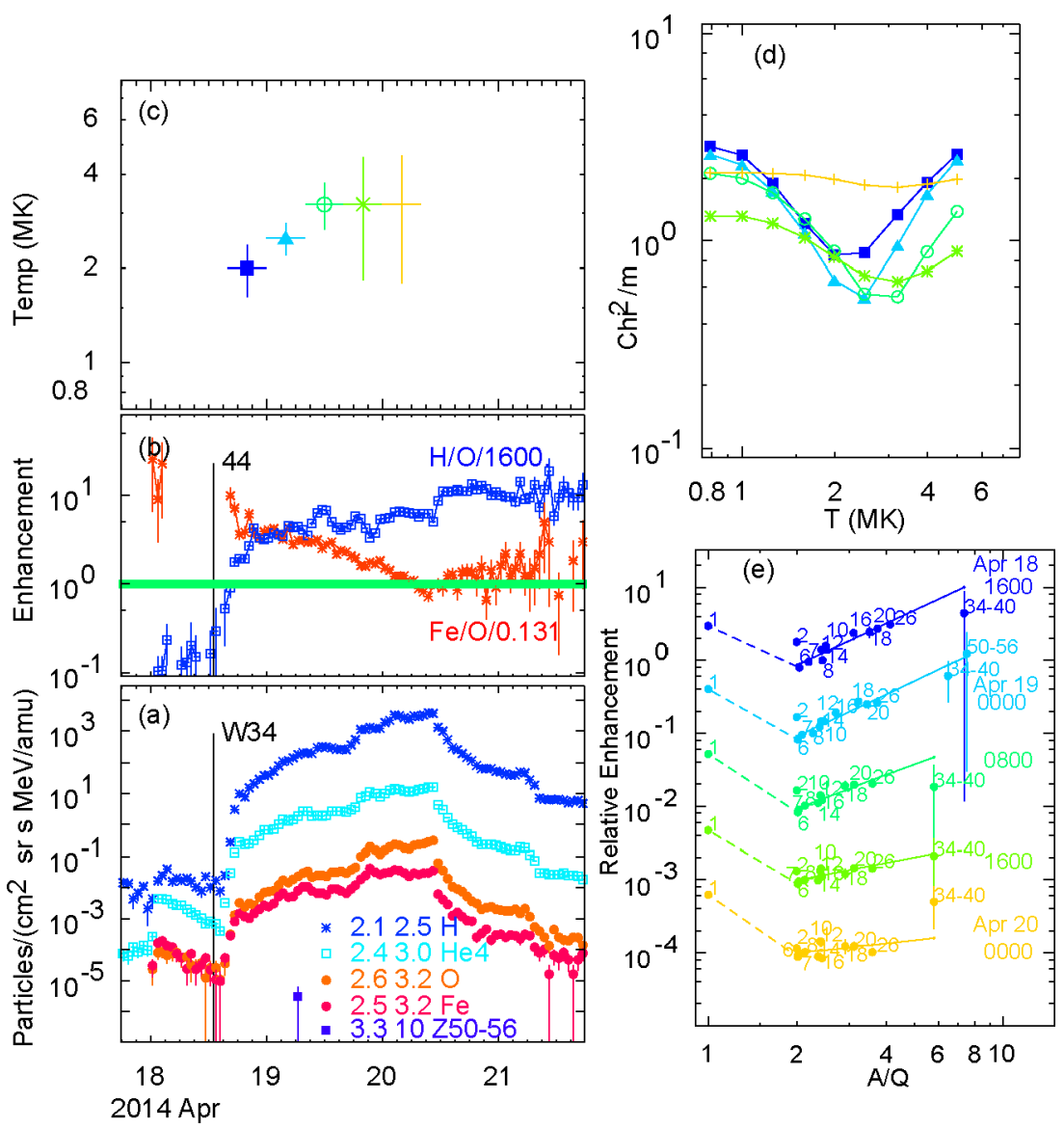

Figure 5 (a) Intensities of $\mathrm{H}, \mathrm{He}, \mathrm{O}, \mathrm{Fe}$, and $50 \leq Z \leq 56$ ions versus time. (b) Normalized abundance enhancements $\mathrm{H} / \mathrm{O}$ and Fe/O versus time. (c) Temperatures versus time for the 18 April 2014 SEP event. (d) Shows $\chi^{2} / m$ versus $T$ for each 8 -hr interval. (e) Shows enhancements, labeled by $Z$, versus $A / Q$ for each 8-hr interval shifted $\times 0.1$, with best-fit power law for elements with $Z \geq 6$ (solid) joined to the enhanced $\mathrm{H}$ by dashed lines. Colors correspond for the six intervals in (c), (d), and (e) and symbols in (c) and (d); times are also listed in (e). Large proton excesses are seen where dashed lines join $\mathrm{H}$ with its associated elements in (e) Event onset is flagged with solar longitude in (a) and event number from Reames (2016a) in (b).

with $T<2 \mathrm{MK}$, not by recycled impulsive material. The gradual SEP events characterized by reaccelerated $T \approx 3 \mathrm{MK}$ ions from prior impulsive events are modest in size in terms of their energetic proton intensities. Perhaps slower shocks are more strongly biased in favor of pre-accelerated suprathermal ions (Laming et al., 2013).

The shock geometry is also a factor in selecting ions that are accelerated from the seed population (Tylka et al., 2005; Tylka and Lee, 2006). When $\theta_{\mathrm{Bn}}$, the angle between the magnetic field and the shock normal, approaches $90^{\circ}$, only the fastest ions can overtake the shock from downstream, so the faster pre-accelerated impulsive ions are favored. Quasiparallel shocks, with $\theta_{\mathrm{Bn}}<45^{\circ}$, are less selective.

It was only possible to assign temperatures to about $70 \%$ of gradual SEP events since at least four reasonably-consistent eight-hour periods were required (Reames, 2016a). Most events that failed this test did so because the dependence on $A / Q$ was too flat, so that the 
Figure 6 The lower panel shows normalized abundances of $\mathrm{O} / \mathrm{C}$ versus $\mathrm{He} / \mathrm{C}$ for 8-hr intervals during gradual SEP events with temperature as the size and color of the points. The upper panels show $\mathrm{Fe} / \mathrm{O}$ versus $\mathrm{He} / \mathrm{C}$ with temperature, proton excess, and $20-\mathrm{MeV}$ proton intensity for each interval highlighted by color and size of the points. Dashed lines indicate reference abundances of $\mathrm{He} / \mathrm{O}=$ 57 and 91.

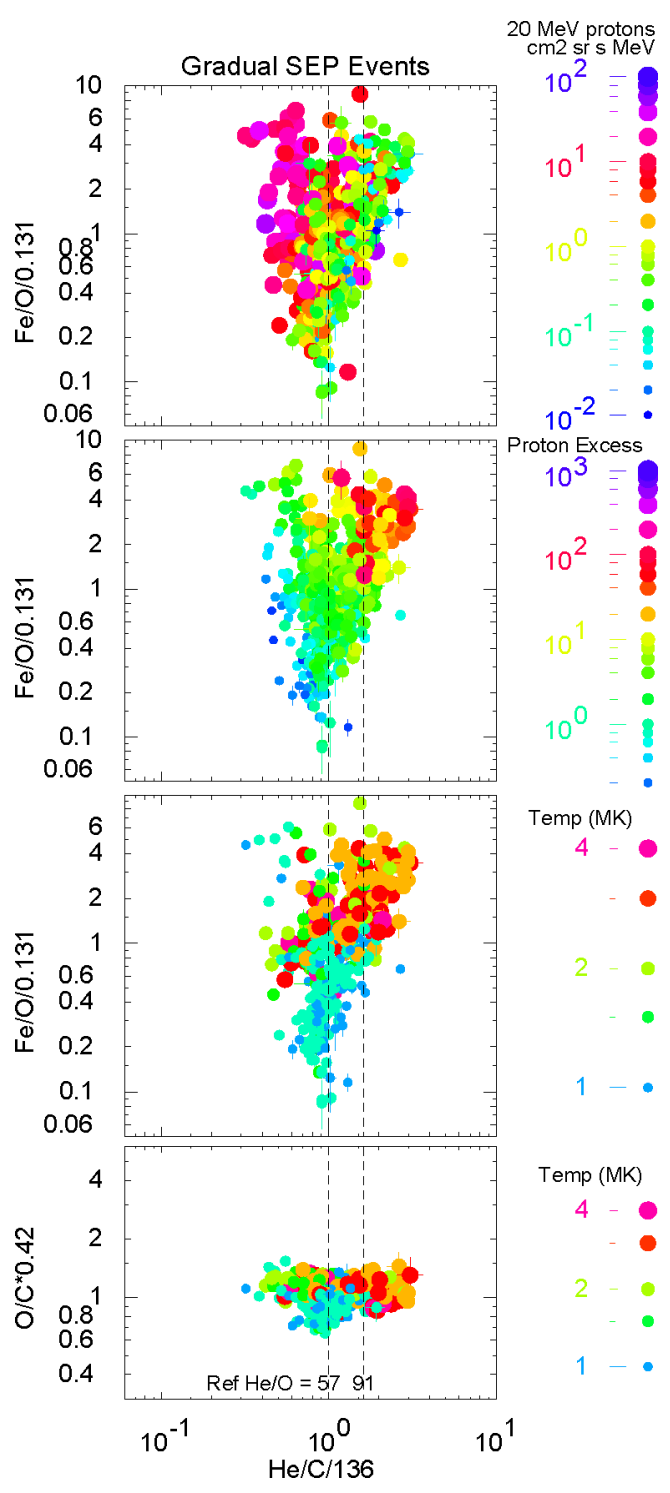

values of $A / Q$, and hence the value of $T$, were indeterminate, i.e. there was no "best" value. Since this occurs when the measured abundances are similar to the reference abundances, these events may be less likely to involve many reaccelerated impulsive suprathermal ions with their strong $A / Q$ enhancements, and may be biased in favor of sampling ambient coronal material.

\section{Comparing CMEs}

We compare some properties of the CMEs associated with gradual and impulsive SEP events in Figure 7. Relevant CME data were found in the SOHO/LASCO CME catalog (Gopal- 
Figure 7 Histograms compare the speed (left panels) and width (right panels) distributions of CMEs associated with impulsive (upper panels) and gradual (lower panels) SEP events. The "?" indicates events with no CME association.

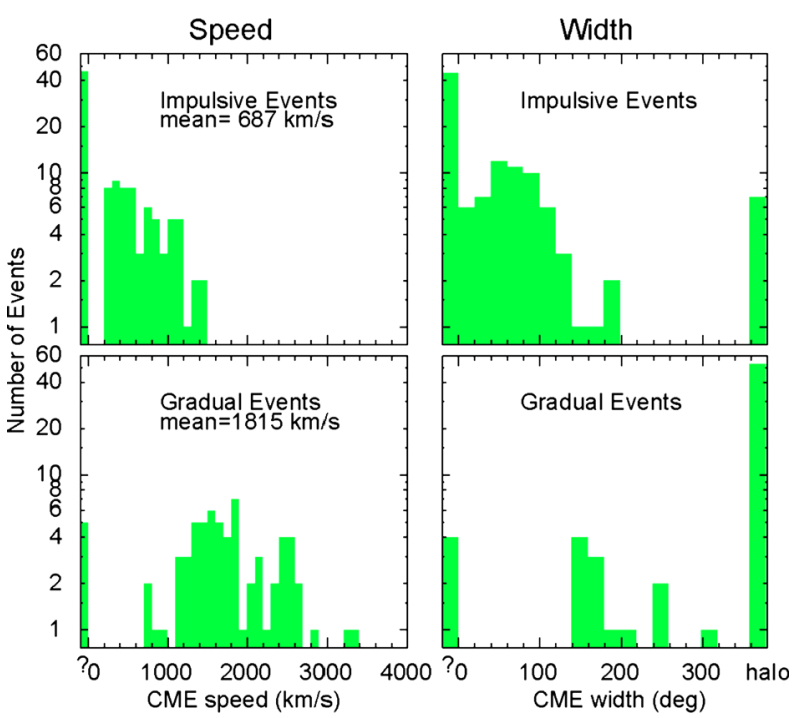

swamy et al., 2009; https://cdaw.gsfc.nasa.gov/CME_list/). The gradual SEP events on our list have wide, mostly halo, fast CMEs with a mean speed of $1815 \mathrm{~km} \mathrm{~s}^{-1}$. The impulsive event list has narrow, mostly $<120^{\circ}$, slow CMEs with a mean speed for these little CMEs of $687 \mathrm{~km} \mathrm{~s}^{-1}$. For many impulsive events, CMEs are too small to be recorded (e.g. Nitta et al., 2006).

Thus, when we distinguish impulsive and gradual events based upon $\mathrm{Fe} / \mathrm{O}$ as shown in Figure 1, we find the associated CMEs have differing properties as shown in Figure 7. The properties of the CMEs associated with impulsive SEP events are those of narrow solar jets, those associated with gradual SEPs are large, wide, eruptive CMEs. This leads us to a suggested explanation of the differences of the width of the distribution of $\mathrm{He} / \mathrm{C}$, for example, shown in the lower panels of Figures 3 and 6, and first suggested by Reames (2016b) and shown in Figure 8. The abundance patterns on the right in Figure 8 are from Reames (2016b). Both populations involve $T \approx 3 \mathrm{MK}$ impulsive SEP ions from magnetic-reconnection sites in solar jets which may be reaccelerated by a shock wave; proton excesses are produced when those shocks also include protons from the ambient coronal material. In the impulsive case, the shock, if any, is produced by the CME from the same event; ions from a single jet reflect local variations in element abundances. In the second case, a large shock from a wide CME sweeps up residual impulsive suprathermal ions from many $(\mathrm{N})$ individual jets that have occurred recently in the vicinity of an entire active region, reducing the abundance fluctuations by a factor of $\sqrt{N}$. The residue from many jets collects in large regions for substantial periods so that ${ }^{3} \mathrm{He}$-rich, Fe-rich background levels are often seen (Desai et al., 2003; Bučík et al., 2014, 2015, 2018a,b; Chen et al., 2015).

Having distinguished differences in the CMEs associated with impulsive and gradual SEP events, we now turn to differences in CMEs that favor different ion seed populations for shock acceleration in gradual SEP events. Figure 6 suggests that the most intense gradual events are associated with cooler plasma while events with the hotter reaccelerated impulsive ions are not so large and intense. Figure 9 shows the timing and CME-speed distributions of ions with differing source-plasma temperatures. Events with the hotter reaccelerated impulsive-SEP source plasma involve somewhat slower, weaker CMEs, probably with quasi-perpendicular shock waves, that tend to arrive early in Solar Cycle 23 and to dominate 


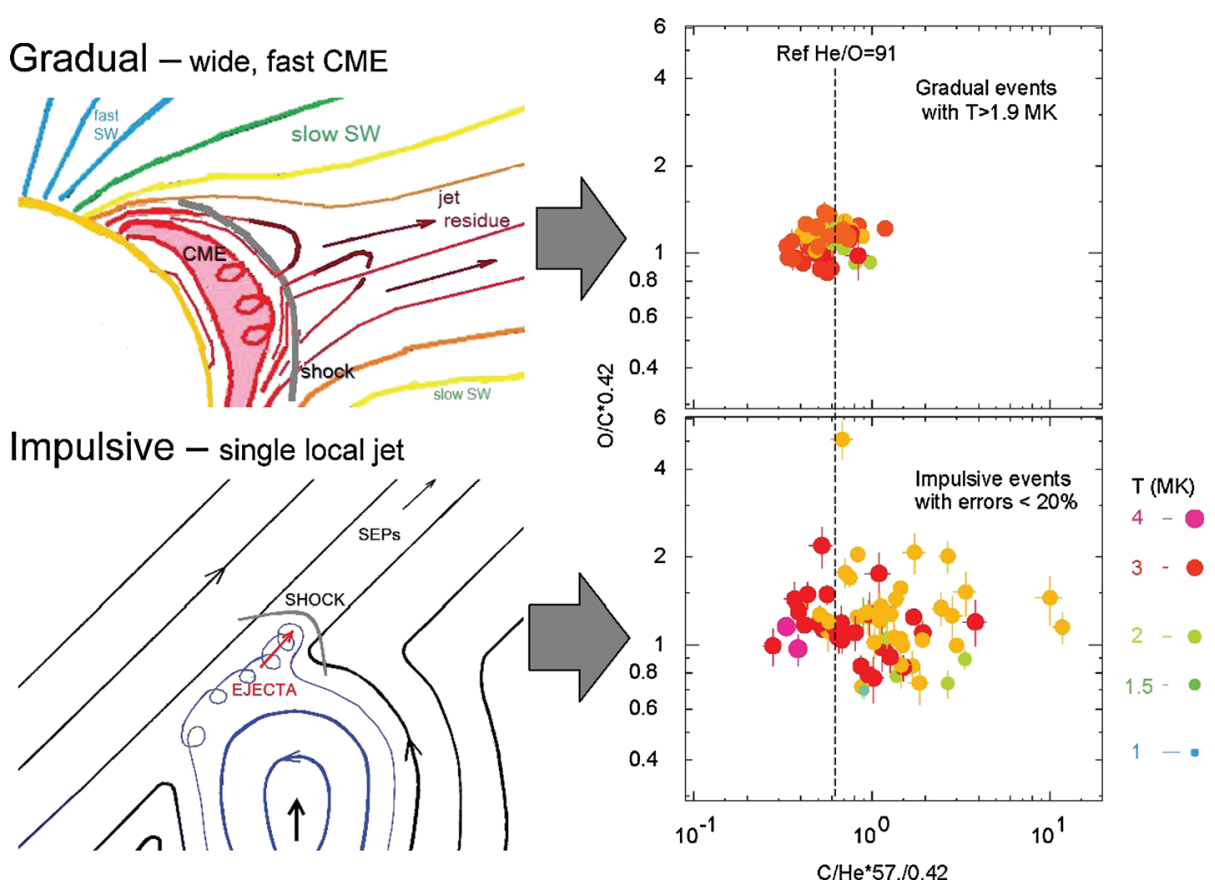

Figure 8 Suggested explanations for the broad distribution of source abundances in impulsive events (below) from variations in single localized jet events where ejecta may or may not drive a shock, and the narrow distribution in high- $T$ gradual events (above) where pre-accelerated impulsive seed populations from many individual jets are averaged by a large shock. Abundance distributions shown (from Reames, 2016b) are equivalent to those that were shown in the lower panels in Figures 3 and 6.

the weak Solar Cycle 24. Faster CMEs that occur later in Cycle 23 tend to be dominated by cooler ambient coronal plasma in which they sample deeply - these more powerful events have less need for pre-accelerated ions. Like SEP events, all solar cycles are not the same.

\section{Discussion}

Can the situation described by Figure 8 explain suppressed He? Models of the FIP effect do suggest reduction in the abundance of He because its high value of FIP makes He ionization and fractionation uniquely slow (Laming, 2009). However, these calculations show suppression by a factor of two, not a factor of ten. However, these calculations involve a quiescent equilibrium flow of plasma across the chromosphere into the corona. What happens when chromospheric material is suddenly thrust up into the corona in a jet as suggested by the emerging-flux model in the lower-left panel of Figure 8? Would it be surprising for more rapid and dynamic FIP processing to lead to a much greater suppression of He? Perhaps partially FIP-processed chromospheric material may suddenly rise to become involved in some He-poor jets. However, while ${ }^{3} \mathrm{He}$ and ${ }^{4} \mathrm{He}$ fractionation should be similar, ${ }^{3} \mathrm{He} /{ }^{4} \mathrm{He}$ tends to be highest when ${ }^{4} \mathrm{He} / \mathrm{C}$ is suppressed.

He suppression is the only FIP-related event-to-event variation we can identify in SEP events. The theory of He suppression (Laming, 2009) also suggests the possible suppression 
Figure 9 Shows the CME speed of events as a function of onset time for gradual SEP events, with source-plasma temperatures indicated by color and size of the points in the lower panel and CME speed distributions for gradual SEP events with $T<1.9 \mathrm{MK}$ and $T>1.9 \mathrm{MK}$ in the middle and upper panels, respectively.
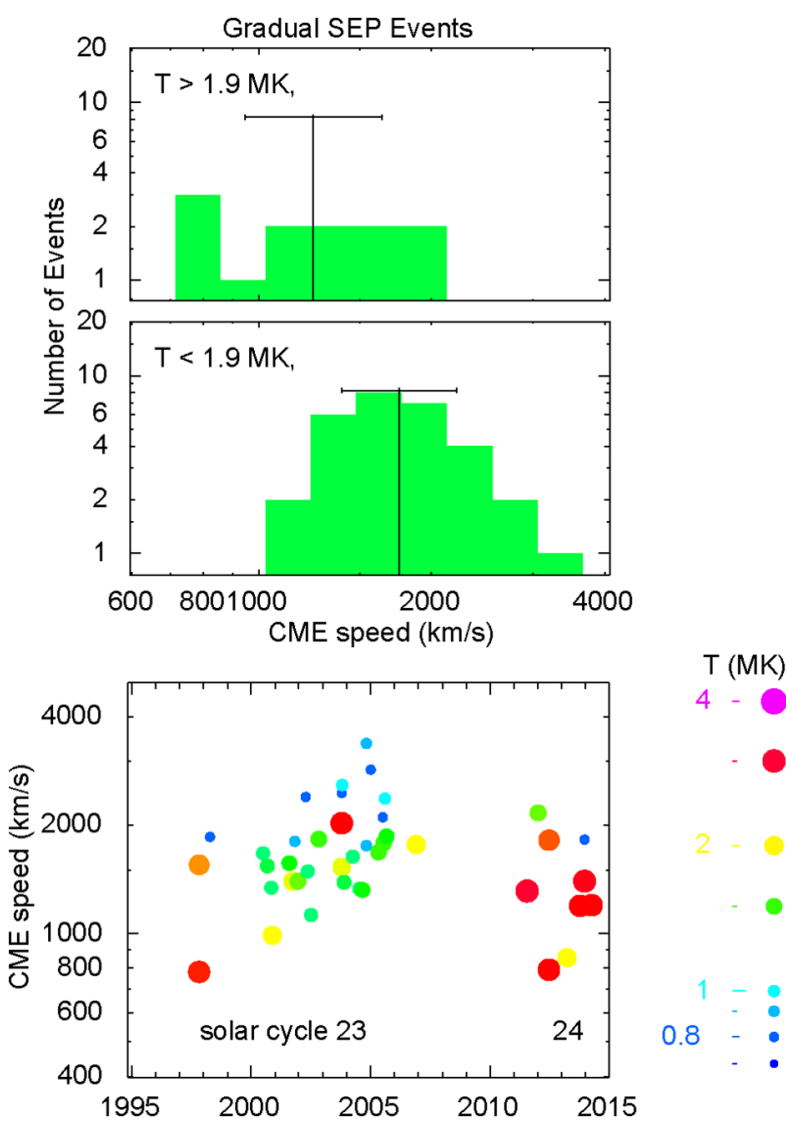

of $\mathrm{Ne}$, but $\mathrm{Ne}$ is nearly always enhanced in impulsive SEP events (see Figure 1), mainly because of the general increase in enhancement with $A / Q$. It is possible that the variations in $\mathrm{O} / \mathrm{C}$ for impulsive events in Figure 8 could occur because $\mathrm{O}$ is not quite fully ionized at 2.5 MK so its value of $A / Q$ may be a bit higher than that of $C$. Naïve researchers occasionally mistake the enhancement in $\mathrm{Fe} / \mathrm{O}$ in impulsive SEP events as a possible FIP effect and a direct measure of the FIP bias, but it is actually part of the 1000-fold power law that stretches from $\mathrm{H}$ to U (see Figure 8 in Reames, Cliver, and Kahler, 2014a; Reames, 2018b). This power law in impulsive SEP events also enhances $\mathrm{Ne} / \mathrm{O}$, for example, where both elements have high FIP. The FIP contribution to the coronal abundances seems to be invariant (as contained in the reference in Table 1), it applies as a coronal basis for all SEP events, and shows no evidence of event-to-event FIP variation other than possibly He. It is important to realize that any FIP-dependent fractionation in impulsive SEP events, including suppression of $\mathrm{He}$, is likely to affect both the SEPs and the ejected plasma from a solar jet; this CME should eventually contribute to the solar wind. Thus FIP-dependent processes could be sought as SEP-SW correlations, although no such correlations have been reported.

The highest source temperatures, $T \approx 3 \mathrm{MK}$ for both impulsive and gradual events, offer the most reliable measure of $\mathrm{He} / \mathrm{O} \approx 90$. This plasma originally comes from the largest jets which sample material at $\approx 1.5$ solar radii (DiFabio et al., 2008). Large gradual events sample cooler ambient plasma at $\geq 2$ solar radii (Reames, 2009a, 2009b), but it is the recycled 
Figure 10 Element

enhancements, labeled by $Z$, versus $A / Q$ for sample impulsive Event 54, with two possible seed-particle sources for shock acceleration from pre-accelerated impulsive ions (blue) and from ambient coronal ions or pre-event plasma (red). Helium is assumed to receive comparable contributions from both sources for this event (Reames, 2019a). Any weak or quasi-perpendicular shock waves may prefer impulsive suprathermal ions at high $Z$ from a two-component seed-particle source.

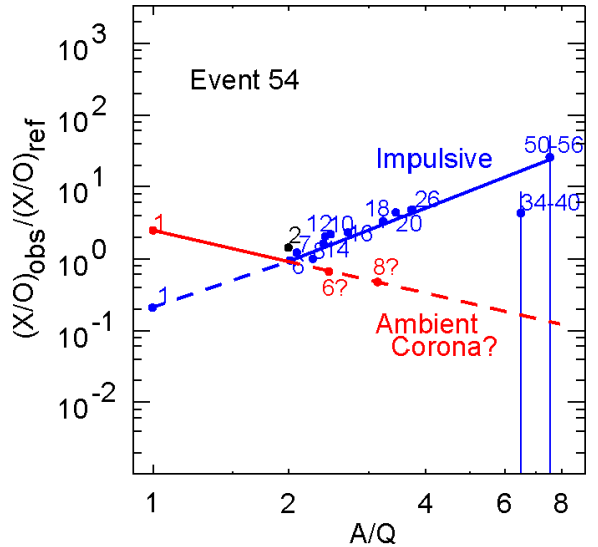

impulsive material in gradual events for which $\mathrm{He} / \mathrm{O} \approx 90$. The overall average value for gradual $\mathrm{SEP}$ events is $\mathrm{He} / \mathrm{O} \approx 57$.

For the smaller "pure" (magnetic reconnection only) impulsive SEP events with no shock reacceleration, and for the largest gradual SEP events which accelerate ambient coronal plasma with $T<2 \mathrm{MK}, \mathrm{H} / \mathrm{O}$ enhancements are generally consistent with power-law fits to the $A / Q$ dependence of abundances of elements with $Z \geq 6$. Any events dominated by shock reacceleration of impulsive suprathermal ions show substantial proton excesses. The only suggested explanation of these proton excesses is shown in Figure 10 where in Event 54 (Reames, Cliver, and Kahler, 2014a; Reames, 2019b) the associated $952 \mathrm{~km} \mathrm{~s}^{-1}$ shock wave primarily selects the enhanced impulsive suprathermal ions which dominate the SEPs with $Z>2$, but protons from the ambient coronal plasma dominate the SEP protons. Abundances of ions with $Z>2$ rise sharply with $A / Q$ because the impulsive suprathermal source ion abundances already increase sharply with $A / Q$, but the ambient ion abundances and, in fact, all accelerated ion abundances, usually decrease modestly with $A / Q$ because of rigidity-dependent transport from the shock. Note that the cooler ambient ions will have lower $Q$ and higher $A / Q$ than the impulsive ions with $T \approx 3 \mathrm{MK}$.

While Figure 10 shows data from an impulsive SEP event, the concept can apply to any event, impulsive or gradual, where a weak or quasi-perpendicular shock wave preferentially reaccelerates residual impulsive suprathermal ions which dominate high $Z$, while protons are mainly sampled from the ambient coronal plasma. However, any ${ }^{4} \mathrm{He}$ accelerated from the ambient plasma in impulsive SEP events will certainly tend to reduce ${ }^{3} \mathrm{He} /{ }^{4} \mathrm{He}$, so impulsive events with proton excesses may not remain ${ }^{3} \mathrm{He}$-rich.

Regarding the general issue of multiple components and the seed population, shock acceleration at corotating interaction regions (CIRs) sometimes shares this complexity (Reames, 2018c). CIRs are formed when high-speed solar-wind streams overtake and collide with slow solar wind emitted earlier in the solar rotation (e.g. Richardson, 2004). This collision leads to two shock waves, a forward shock propagating outward into the slow wind and a stronger reverse shock propagating sunward into the fast wind. Usually these shocks form outside $1 \mathrm{AU}$ and strengthen out to $5 \mathrm{AU}$ (Van Hollebeke, McDonald, and von Rosenvinge, 1978). Low-rigidity ions tend to be retained near the shock while high-rigidity ions spread widely (e.g. Reames et al., 1997). To first order, the only source of particles available for acceleration at CIR shocks is the solar wind, and the energetic particles at CIRs typically show solar-wind abundances near the shocks and abundances that decrease as power laws in $A / Q$ more and more steeply with distance from the shock (Reames, 2018c). However, seed 
particles, or simply background, from SEP events, especially small impulsive SEP events, can inject Fe-rich material that easily dominates the otherwise-suppressed abundance of $\mathrm{Fe}$ from the solar wind, but contributes much less at lower $Z$ (Reames, 2018c). This is reminiscent of the pattern shown in Figure 10. These multi-component events tended to hide the $A / Q$ dependence and complicated the source composition in early measurements of CIR events, especially for the weaker shocks associated with slower solar-wind streams.

In SEP events, the rigidity dependence that leads to the $A / Q$ dependence can come from several factors. The acceleration of ions in islands of magnetic reconnection leads directly to power laws in $A / Q$ when the ions undergo Fermi acceleration as they are reflected back and forth from the ends of collapsing magnetic islands (Drake et al., 2009). Scattering during transit along magnetic fields also depends upon $A / Q$ as noted above (Parker, 1963; Ng, Reames, and Tylka, 2003; Reames, 2016a, 2016b, 2019b). In shocks, the dominant protons play a special role, not only are they spectators, resonantly scattering from existing waves like all other ions, but they can generate or amplify resonant waves as they stream away from the shock, increasing the scattering and acceleration of ions that follow (Bell, 1978; Lee, 1983, 2005; Ng and Reames, 2008; Reames and Ng, 2010). The wave number of resonant Alfvén waves is $k \approx B / \mu P$, where $B$ is the magnetic field strength, $\mu$ is the cosine of the ion's pitch angle with the field, and $P$ is the ion's rigidity. Thus, if we assume $\mu \approx 1$ for simplicity, $2.5 \mathrm{MeV}$ protons are scattered by self-generated waves at $2.5 \mathrm{MeV}$, for example, but $2.5 \mathrm{MeV} \mathrm{amu}^{-1} \mathrm{He}, \mathrm{C}$, or $\mathrm{O}$ with $A / Q=2$ (at $T \approx 3 \mathrm{MK}$ ) resonate with waves generated by $10-\mathrm{MeV}$ protons, and $2.5 \mathrm{MeV} \mathrm{amu}^{-1} \mathrm{Fe}$ at $A / Q=4$ resonates with waves generated by streaming $39-\mathrm{MeV}$ protons. A pure power-law dependence of enhancements upon $A / Q$ may require a power-law proton rigidity spectrum. Differences in resonance can produce a proton excess early in a gradual event when, for example, $2.5 \mathrm{MeV}$ protons begin to arrive, as yet unscattered, while $2.5 \mathrm{MeV} \mathrm{He}$ at the same time has been scattered and retarded by traversing waves generated by $10-\mathrm{MeV}$ protons that arrived much earlier, increasing $\mathrm{H} / \mathrm{He}$ (Reames, Ng, and Tylka, 2000). The understanding of SEP events could be greatly enhanced by improved theory and modeling of time-dependent generation of resonant waves by protons and the coupling of those waves to other ions. While the full time-dependent evolution of proton and wave spectra at shock waves have been modeled in some detail (e.g. $\mathrm{Ng}$ and Reames, 2010), the co-evolution of the spectra of other ions has not yet been included.

\section{Summary}

The study of the $A / Q$ dependence of element abundance enhancements has added a new approach to the study of SEP events. Approximate power-law dependence has led to determination of best-fit values of $Q$ and estimates of the underlying source-plasma temperatures. These temperatures distinguish ions enhanced during magnetic reconnection on open field lines in solar jets $(T \approx 3 \mathrm{MK})$ from ions of the cooler $(<2 \mathrm{MK})$ ambient coronal plasma. Adding $\mathrm{H}$ and $\mathrm{He}$ to these abundance studies provides important new information and suggests four categories of acceleration of SEPs:

i) "Pure," shock-free impulsive SEP events accelerate ions in islands of magnetic reconnection in solar jets. Element abundance enhancements increase as a power law in $A / Q$ from $\mathrm{H}$ to heavy elements like $\mathrm{Pb}$; they can be distinguished by $\mathrm{Fe} / \mathrm{O}$ that is over four times the coronal abundance. He/O may be greatly suppressed in occasional events by a rapid rise of material that is too fast to allow much ionization of high-FIP He. Electrons streaming out from the event generate waves that are resonantly absorbed to enhance 
${ }^{3} \mathrm{He}$; these electrons produce a type III radio burst. Plasma may also be ejected from the event, producing a narrow CME that is too slow to drive a shock wave.

ii) An impulsive + shock event occurs when the narrow CME from a jet, that of an otherwise pure impulsive event, is fast enough to drive a shock wave. The shock wave samples all available ions, those from the ambient plasma and residual energetic ions from the pure event. The abundant ambient protons form the wave structure at the shock and dominate at $Z=1$, but the pre-enhanced, pre-accelerated ions are selected by the weak shock and dominate the $Z>2$ region. This appears as a large proton excess.

iii) A fast, wide CME from an eruptive event drives a fast shock wave that expands broadly, producing an energetic gradual SEP event that lasts many days. If the shock is quasiparallel or samples deeply into the tail of the thermal distribution of the ambient plasma with $T<2 \mathrm{MK}$, it will produce a "pure" gradual event with essentially coronal ion abundances modified by a power-law dependence on $A / Q$ that may be enhanced or suppressed during ion transport. Protons generally fit with other ions although some regions of unusual transport may produce modest local excesses or depletions of protons. Any impulsive suprathermal ions present are also accelerated by the shock, but their contribution is overwhelmed but the accelerated ambient coronal ions; these events do not need pre-accelerated ions.

iv) A fast, wide CME from an eruptive event drives a moderately fast shock wave producing a gradual SEP event. The shock may be quasi-perpendicular (or just weak) so its final contribution from sampling of ambient plasma is limited mainly to protons, while preferring residual impulsive suprathermal ions surviving from a dozen or so earlier impulsive events that combine to produce well-defined average impulsive-SEP abundances for $Z>2$.

Thus, we still think impulsive SEP events are produced in solar jets, initially from magnetic reconnection, and ions in gradual SEP events are still swept up and accelerated by shock waves driven by wide, fast CMEs. However, weak or quasi-perpendicular shock waves in either event class manage to accelerate only significant protons from the ambient coronal plasma, but preferentially recycle pre-accelerated impulsive-SEP ions, with their built-in abundance bias, at higher $Z$.

The study of power-law patterns of element abundance enhancements has given us new parameters to better distinguish and organize physical processes in the large sample of SEP events we have seen. Soon the Wind spacecraft will enter its third solar cycle; will the abundances of the new SEP events return to the pattern of Solar Cycle 23, as we would predict, or will they differ entirely?

Acknowledgement CME data were taken from the CDAW LASCO catalog. This CME catalog is generated and maintained at the CDAW Data Center by NASA and The Catholic University of America in cooperation with the Naval Research Laboratory.

Disclosure of Potential Conflicts of Interest The author declares he has no conflicts of interest.

Publisher's Note Springer Nature remains neutral with regard to jurisdictional claims in published maps and institutional affiliations.

Open Access This article is distributed under the terms of the Creative Commons Attribution 4.0 International License (http://creativecommons.org/licenses/by/4.0/), which permits unrestricted use, distribution, and reproduction in any medium, provided you give appropriate credit to the original author(s) and the source, provide a link to the Creative Commons license, and indicate if changes were made. 
Table 1 Reference SEP abundances, photospheric, and impulsive SEP abundances are compared for various elements.

\begin{tabular}{llllll}
\hline & $Z$ & $\begin{array}{l}\text { FIP } \\
{[\mathrm{eV}]}\end{array}$ & Photosphere $^{\mathrm{a}}$ & $\begin{array}{l}\text { Reference } \\
\mathrm{SEPs}^{\mathrm{b}}\end{array}$ & $\begin{array}{l}\text { Avg. Impulsive } \\
\text { SEPs }^{\mathrm{c}}\end{array}$ \\
\hline $\mathrm{H}$ & 1 & 13.6 & $(1.74 \pm 0.17) \times 10^{6 \mathrm{~d}}$ & $(1.6 \pm 0.2) \times 10^{6}$ & - \\
$\mathrm{He}$ & 2 & 24.6 & $146000 \pm 6700$ & $57000 \pm 5000$ & $53000 \pm 3000^{\mathrm{e}}$ \\
$\mathrm{C}$ & 6 & 11.3 & $550 \pm 76^{\mathrm{d}}$ & $420 \pm 10$ & $386 \pm 8$ \\
$\mathrm{~N}$ & 7 & 14.5 & $126 \pm 35^{\mathrm{d}}$ & $128 \pm 8$ & $139 \pm 4$ \\
$\mathrm{O}$ & 8 & 13.6 & $1000 \pm 161^{\mathrm{d}}$ & $1000 \pm 10$ & $1000 \pm 10$ \\
$\mathrm{Ne}$ & 10 & 21.6 & $195 \pm 45$ & $157 \pm 10$ & $478 \pm 24$ \\
$\mathrm{Mg}$ & 12 & 7.6 & $60.3 \pm 8.3$ & $178 \pm 4$ & $404 \pm 30$ \\
$\mathrm{Si}$ & 14 & 8.2 & $57.5 \pm 8$ & $151 \pm 4$ & $325 \pm 12$ \\
$\mathrm{~S}$ & 16 & 10.4 & $25.1 \pm 2.9^{\mathrm{d}}$ & $25 \pm 2$ & $84 \pm 4$ \\
$\mathrm{Ar}$ & 18 & 15.8 & $5.5 \pm 1.3$ & $4.3 \pm 0.4$ & $34 \pm 2$ \\
$\mathrm{Ca}$ & 20 & 6.1 & $3.7 \pm 0.6$ & $11 \pm 1$ & $85 \pm 4$ \\
$\mathrm{Fe}$ & 26 & 7.9 & $57.5 \pm 8.0^{\mathrm{d}}$ & $131 \pm 6$ & $1170 \pm 48$ \\
$\mathrm{Se}-\mathrm{Zr}$ & $34-40$ & - & $\approx 0.0118$ & $0.04 \pm 0.01$ & $2.0 \pm 0.2$ \\
$\mathrm{Sn}-\mathrm{Ba}$ & $50-56$ & - & $\approx 0.00121$ & $0.0066 \pm 0.001$ & $2.0 \pm 2$ \\
$\mathrm{Os}-\mathrm{Pb}$ & $76-82$ & - & $\approx 0.00045$ & $0.0007 \pm 0.0003$ & $0.64 \pm 0.12$ \\
\hline
\end{tabular}

${ }^{\text {a}}$ Lodders, Palme, and Gail (2009); see also Asplund et al. (2009).

${ }^{\mathrm{b}}$ Reames (1995a, 2014, 2017a).

${ }^{\mathrm{c}}$ Reames, Cliver, and Kahler (2014a).

${ }^{\mathrm{d}}$ Caffau et al. (2011).

${ }^{\mathrm{e}}$ Reames (2019a).

\section{Appendix: Reference Abundances of Elements}

The average element abundances in gradual SEP events are a measure of the coronal abundances sampled by SEP events (Reference SEPs in Table 1). They differ from photospheric abundances in Table 1 by a factor which depends upon FIP (e.g. Reames, 2018a, 2018b; Laming et al., 2019). More complete tables of SEP abundances of 21 elements that include rarer species are given by Reames (2017a, 2018a). Ion "enhancements" are defined as the observed abundance of a species, relative to $\mathrm{O}$, divided by the reference abundance of that species, relative to $\mathrm{O}$.

\section{References}

Asplund, M., Grevesse, N., Sauval, A.J., Scott, P.: 2009, The chemical composition of the sun. Annu. Rev. Astron. Astrophys. 47, 481. DOI.

Bell, A.R.: 1978, The acceleration of cosmic rays in shock fronts. I. Mon. Not. Roy. Astron. Soc. 182, 147. DOI.

Breneman, H.H., Stone, E.C.: 1985, Solar coronal and photospheric abundances from solar energetic particle measurements. Astrophys. J. Lett. 299, L57. DOI.

Bučík, R., Innes, D.E., Mall, U., Korth, A., Mason, G.M., Gómez-Herrero, R.: 2014, Multi-spacecraft observations of recurrent ${ }^{3} \mathrm{He}$-rich solar energetic particles. Astrophys. J. 786, 71. DOI.

Bučík, R., Innes, D.E., Chen, N.H., Mason, G.M., Gómez-Herrero, R., Wiedenbeck, M.E.: 2015, Long-lived energetic particle source regions on the Sun. J. Phys. Conf. Ser. 642, 012002. DOI. 
Bučík, R., Innes, D.E., Mason, G.M., Wiedenbeck, M.E., Gómez-Herrero, R., Nitta, N.V.: 2018a, ${ }^{3}$ He-rich solar energetic particles in helical jets on the Sun. Astrophys. J. 852, 76. DOI.

Bučík, R., Wiedenbeck, M.E., Mason, G.M., Gómez-Herrero, R., Nitta, N.V., Wang, L.: 2018b, ${ }^{3}$ He-rich solar energetic particles from sunspot jets. Astrophys. J. Lett. 869, L21. DOI.

Caffau, E., Ludwig, H.-G., Steffen, M., Freytag, B., Bonofacio, P.: 2011, Solar chemical abundances determined with a CO5BOLD 3D model atmosphere. Solar Phys. 268, 255. DOI.

Cane, H.V., Reames, D.V., von Rosenvinge, T.T.: 1988, The role of interplanetary shocks in the longitude distribution of solar energetic particles. J. Geophys. Res. 93, 9555. DOI.

Chen, N.H., Bučík, R., Innes, D.E., Mason, G.M.: 2015, Case studies of multi-day ${ }^{3}$ He-rich solar energetic particle periods. Astron. Astrophys. 580, 16. DOI.

Cliver, E.W., Kahler, S.W., Reames, D.V.: 2004, Coronal shocks and solar energetic proton events. Astrophys. J. 605, 902. DOI.

Desai, M.I., Giacalone, J.: 2016, Large gradual solar energetic particle events. Living Rev. Solar Phys. DOI.

Desai, M.I., Mason, G.M., Dwyer, J.R., Mazur, J.E., Gold, R.E., Krimigis, S.M., Smith, C.W., Skoug, R.M.: 2003, Evidence for a suprathermal seed population of heavy ions accelerated by interplanetary shocks near 1 AU. Astrophys. J. 588, 1149. DOI.

DiFabio, R., Guo, Z., Möbius, E., Klecker, B., Kucharek, H., Mason, G.M., Popecki, M.: 2008, Energydependent charge states and their connection with ion abundances in impulsive solar energetic particle events. Astrophys. J. 687, 623. DOI.

Drake, J.F., Cassak, P.A., Shay, M.A., Swisdak, M., Quataert, E.: 2009, A magnetic reconnection mechanism for ion acceleration and abundance enhancements in impulsive flares. Astrophys. J. Lett. 700, L16. DOI.

Gopalswamy, N., Yashiro, S., Michalek, G., Stenborg, G., Vourlidas, A., Freeland, S., Howard, R.: 2009, The SOHO/LASCO CME catalog. Earth Moon Planets 104, 295. DOI.

Gopalswamy, N., Xie, H., Yashiro, S., Akiyama, S., Mäkelä, P., Usoskin, I.G.: 2012, Properties of ground level enhancement events and the associated solar eruptions during solar cycle 23. Space Sci. Rev. 171, 23. DOI.

Gosling, J.T.: 1993, The solar flare myth. J. Geophys. Res. 98, 18937. DOI.

Kahler, S.W.: 2001, The correlation between solar energetic particle peak intensities and speeds of coronal mass ejections: effects of ambient particle intensities and energy spectra. J. Geophys. Res. 106, 20947. DOI.

Kahler, S.W., Reames, D.V., Sheeley, N.R. Jr.: 2001, Coronal mass ejections associated with impulsive solar energetic particle events. Astrophys. J. 562, 558. DOI.

Kahler, S.W., Tylka, A.J., Reames, D.V.: 2009, A comparison of elemental abundance ratios in SEP events in fast and slow solar-wind regions. Astrophys. J. 701, 561. DOI.

Kahler, S.W., Sheeley, N.R. Jr., Howard, R.A., Koomen, M.J., Michels, D.J.: 1984, Associations between coronal mass ejections and solar energetic proton events. J. Geophys. Res. 89, 9683. DOI.

Kouloumvakos, A., Rouillard, A.P., Wu, Y., Vainio, R., Vourlidas, A., Plotnikov, I., Afanasiev, A., Önel, H.: 2019, Connecting the properties of coronal shock waves with those of solar energetic particles. Astrophys. J. 876, 80. DOI.

Laming, J.M.: 2009, Non-WKB models of the first ionization potential effect: implications for solar coronal heating and the coronal helium and neon abundances. Astrophys. J. 695, 954. DOI.

Laming, J.M.: 2015, The FIP and inverse FIP effects in solar and stellar coronae. Living Rev. Solar Phys. 12, 2. DOI.

Laming, J.M., Moses, J.D., Ko, Y.-K., Ng, C.K., Rakowski, C.E., Tylka, A.J.: 2013, On the remote detection of suprathermal ions in the solar corona and their role as seeds for solar energetic particle production. Astrophys. J. 770, 73. DOI.

Laming, J.M., Vourlidas, A., Korendyke, C., Chua, D, Cranmer, S.R., Ko, Y.-K.: 2019, Element abundances: a new diagnostic for the solar wind. Astrophys. J. 879, 124. DOI.

Lee, M.A.: 1983, Coupled hydromagnetic wave excitation and ion acceleration at interplanetary traveling shocks. J. Geophys. Res. 88, 6109. DOI.

Lee, M.A.: 2005, Coupled hydromagnetic wave excitation and ion acceleration at an evolving coronal/interplanetary shock. Astrophys. J. Suppl. 158, 38. DOI.

Lee, M.A., Mewaldt, R.A., Giacalone, J.: 2012, Shock acceleration of ions in the heliosphere. Space Sci. Rev. 173, 247. DOI.

Lodders, K., Palme, H., Gail, H.-P.: 2009, Abundances of the elements in the solar system. In: Trümper, J.E. (ed.) Landolt-Börnstein, New Series, VI/4B, Springer, Berlin, 560. Chapter 4.4.

Mason, G.M.: 2007, ${ }^{3}$ He-rich solar energetic particle events. Space Sci. Rev. 130, 231. DOI.

Mason, G.M., Mazur, J.E., Dwyer, J.R.: 2002, A new heavy ion abundance enrichment pattern in ${ }^{3} \mathrm{He}-\mathrm{rich}$ solar particle events. Astrophys. J. Lett. 565, L51. DOI. 
Mason, G.M., Mazur, J.E., Dwyer, J.R., Jokippi, J.R., Gold, R.E., Krimigis, S.M.: 2004, Abundances of heavy and ultraheavy ions in ${ }^{3} \mathrm{He}$-rich solar flares. Astrophys. J. 606, 555. DOI.

Mewaldt, R.A., Cohen, C.M.S., Leske, R.A., Christian, E.R., Cummings, A.C., Stone, E.C., von Rosenvinge, T.T., Wiedenbeck, M.E.: 2002, Fractionation of solar energetic particles and solar wind according to first ionization potential. Adv. Space Res. 30, 79. DOI.

Meyer, J.-P.: 1985, The baseline composition of solar energetic particles. Astrophys. J. Suppl. 57, 151. DOI.

Ng, C.K., Reames, D.V.: 2008, Shock acceleration of solar energetic protons: the first 10 minutes. Astrophys. J. Lett. 686, L123. DOI.

Ng, C.K., Reames, D.V., Tylka, A.J.: 1999, Effect of proton-amplified waves on the evolution of solar energetic particle composition in gradual events. Geophys. Res. Lett. 26, 2145. DOI.

Ng, C.K., Reames, D.V., Tylka, A.J.: 2001, Abundances, spectra, and anisotropies in the 1998 Sep 30 and 2000 Apr 4 large SEP events. In: Proc. 27th Int. Cosmic-Ray Conf., Hamburg, 8, 3140.

Ng, C.K., Reames, D.V., Tylka, A.J.: 2003, Modeling shock-accelerated solar energetic particles coupled to interplanetary Alfvén waves. Astrophys. J. 591, 461. DOI.

Ng, C.K., Reames, D.V., Tylka, A.J.: 2012, Solar energetic particles: shock acceleration and transport through self-amplified waves. AIP Conf. Proc. 1436, 212. DOI.

Nitta, N.V., Reames, D.V., DeRosa, M.L., Yashiro, S., Gopalswamy, N.: 2006, Solar sources of impulsive solar energetic particle events and their magnetic field connection to the Earth. Astrophys. J. 650, 438. DOI.

Parker, E.N.: 1963, Interplanetary Dynamical Processes, Interscience, New York.

Reames, D.V.: 1988, Bimodal abundances in the energetic particles of solar and interplanetary origin. Astrophys. J. Lett. 330, L71. DOI.

Reames, D.V.: 1995a, Coronal abundances determined from energetic particles. Adv. Space Res. 15(7), 41.

Reames, D.V.: 1995b, Solar energetic particles: a paradigm shift. Rev. Geophys. 33(Suppl.), 585. DOI.

Reames, D.V.: 1999, Particle acceleration at the sun and in the heliosphere. Space Sci. Rev. 90, 413. DOI.

Reames, D.V.: 2000, Abundances of trans-iron elements in solar energetic particle events. Astrophys. J. Lett. 540, L111. DOI.

Reames, D.V.: 2009a, Solar release times of energetic particles in ground-level events. Astrophys. J. 693, 812. DOI.

Reames, D.V.: 2009b, Solar energetic-particle release times in historic ground-level events. Astrophys. J. 706, 844. DOI.

Reames, D.V.: 2013, The two sources of solar energetic particles. Space Sci. Rev. 175, 53. DOI.

Reames, D.V.: 2014, Element abundances in solar energetic particles and the solar corona. Solar Phys. 289, 977. DOI.

Reames, D.V.: 2015, What are the sources of solar energetic particles? Element abundances and source plasma temperatures. Space Sci. Rev. 194, 303. DOI.

Reames, D.V.: 2016a, Temperature of the source plasma in gradual solar energetic particle events. Solar Phys. 291, 911. DOI.

Reames, D.V.: 2016b, The origin of element abundance variations in solar energetic particles. Solar Phys. 291, 2099. DOI.

Reames, D.V.: 2017a, Solar Energetic Particles, Lec. Notes Phys. 932, Springer, Berlin. DOI. ISBN 978-3319-50870-2

Reames, D.V.: 2017b, The abundance of helium in the source plasma of solar energetic particles. Solar Phys. 292, 156. DOI. arXiv.

Reames, D.V.: 2018a, The "FIP effect" and the origins of solar energetic particles and of the solar wind. Solar Phys. 293, 47. DOI. arXiv.

Reames, D.V.: 2018b, Abundances, ionization states, temperatures, and FIP in solar energetic particles. Space Sci. Rev. 214, 61. DOI. arXiv.

Reames, D.V.: 2018c, Corotating shock waves and the solar-wind source of energetic ion abundances: power laws in $A / Q$. Solar Phys. 293, 144. DOI. arXiv.

Reames, D.V.: 2019a, Helium suppression in impulsive solar energetic-particle events. Solar Phys. $294,32$. DOI. arXiv.

Reames, D.V.: 2019b, Hydrogen and the abundances of elements in impulsive solar energetic-particle events. Solar Phys. 294, 37. DOI.

Reames, D.V.: 2019c, Hydrogen and the abundances of elements in gradual solar energetic-particle events. Solar Phys. 294, 69. DOI.

Reames, D.V., Barbier, L.M., Ng, C.K.: 1996, The spatial distribution of particles accelerated by coronal mass ejection-driven shocks. Astrophys. J. 466, 473. DOI.

Reames, D.V., Cliver, E.W., Kahler, S.W.: 2014a, Abundance enhancements in impulsive solar energeticparticle events with associated coronal mass ejections. Solar Phys. 289, 3817. DOI. 
Reames, D.V., Cliver, E.W., Kahler, S.W.: 2014b, Variations in abundance enhancements in impulsive solar energetic-particle events and related CMEs and flares. Solar Phys. 289, 4675. DOI.

Reames, D.V., Cliver, E.W., Kahler, S.W.: 2015, Temperature of the source plasma for impulsive solar energetic particles. Solar Phys. 290, 1761. DOI.

Reames, D.V., Meyer, J.P., von Rosenvinge, T.T.: 1994, Energetic-particle abundances in impulsive solar flare events. Astrophys. J. Suppl. 90, 649. DOI.

Reames, D.V., Ng, C.K.: 2004, Heavy-element abundances in solar energetic particle events. Astrophys. J. 610, 510. DOI.

Reames, D.V., Ng, C.K.: 2010, Streaming-limited intensities of solar energetic particles on the intensity plateau. Astrophys. J. 723, 1286. DOI.

Reames, D.V., Ng, C.K., Tylka, A.J.: 2000, Initial time dependence of abundances in solar particle events. Astrophys. J. Lett. 531, L83. DOI.

Reames, D.V., Stone, R.G.: 1986, The identification of solar ${ }^{3} \mathrm{He}$-rich events and the study of particle acceleration at the sun. Astrophys. J. 308, 902. DOI.

Reames, D.V., von Rosenvinge, T.T., Lin, R.P.: 1985, Solar ${ }^{3}$ He-rich events and nonrelativistic electron events - a new association. Astrophys. J. 292, 716. DOI.

Reames, D.V., Ng, C.K., Mason, G.M., Dwyer, J.R., Mazur, E.J., von Rosenvinge, T.T.: 1997, Late-phase acceleration of energetic ions in corotating interaction regions. Geophys. Res. Lett. 24, 2917. DOI.

Richardson, I.G.: 2004, Energetic particles and corotating interaction regions in the solar wind. Space Sci. Rev. 111, 267. DOI.

Rouillard, A., Sheeley, N.R. Jr., Tylka, A., Vourlidas, A., Ng, C.K., Rakowski, C., Cohen, C.M.S., Mewaldt, R.A., Mason, G.M., Reames, D., et al.: 2012, The longitudinal properties of a solar energetic particle event investigated using modern solar imaging. Astrophys. J. 752, 44. DOI.

Serlemitsos, A.T., Balasubrahmanyan, V.K.: 1975, Solar particle events with anomalously large relative abundance of ${ }^{3}$ He. Astrophys. J. 198, 195. DOI.

Temerin, M., Roth, I.: 1992, The production of ${ }^{3} \mathrm{He}$ and heavy ion enrichment in ${ }^{3} \mathrm{He}$-rich flares by electromagnetic hydrogen cyclotron waves. Astrophys. J. Lett. 391, L105. DOI.

Tylka, A.J., Lee, M.A.: 2006, Spectral and compositional characteristics of gradual and impulsive solar energetic particle events. Astrophys. J. 646, 1319. DOI.

Tylka, A.J., Cohen, C.M.S., Deitrich, W.F., Maclennan, C.G., McGuire, R.E., Ng, C.K., Reames, D.V.: 2001, Evidence for remnant flare suprathermals in the source population of solar energetic particles in the 2000 Bastille Day event. Astrophys. J. Lett. 558, L59. DOI.

Tylka, A.J., Cohen, C.M.S., Dietrich, W.F., Lee, M.A., Maclennan, C.G., Mewaldt, R.A., Ng, C.K., Reames, D.V.: 2005, Shock geometry, seed populations, and the origin of variable elemental composition at high energies in large gradual solar particle events. Astrophys. J. 625, 474. DOI.

Van Hollebeke, M.A.I., McDonald, F.B., von Rosenvinge, T.T.: 1978, The radial variation of corotating energetic particle streams in the inner and outer solar system. J. Geophys. Res. 83, 4723. DOI.

von Rosenvinge, T.T., Barbier, L.M., Karsch, J., Liberman, R., Madden, M.P., Nolan, T., Reames, D.V., Ryan, L., Singh, S., Trexel, H.: 1995, The energetic particles: acceleration, composition, and transport (EPACT) investigation on the wind spacecraft. Space Sci. Rev. 71, 152. DOI.

Webber, W.R.: 1975, Solar and galactic cosmic ray abundances - a comparison and some comments. In: Proc. 14th Int. Cosmic Ray Conf., Munich 5, 1597.

Zank, G.P., Rice, W.K.M., Wu, C.C.: 2000, Particle acceleration and coronal mass ejection driven shocks: a theoretical model. J. Geophys. Res. 105, 25079. DOI. 